\title{
Towards an Intelligent Architecture Creating Adaptive Building Systems for Inhabitation
}

\author{
$>$ Andrew John Wit \\ Ball State University, U.S.A \\ a@wit-o.us
}

\begin{abstract}
Existing typologies of emergency housing rely heavily on conventional designs, materials and labor-intensive construction methodologies, which in post-disaster environments place large amounts of strain on the surrounding communities, material manufacturers and financial systems. With ever more unpredictable environmental conditions, should our new housing prototypes not also have the ability to simultaneously adapt to rapidly changing environmental conditions? This paper investigates the potential of developing a new typology of rapidly deployable emergency housing prototypes through the creation of a system which relies on embedded design intelligence, advanced fabrication and adaptable systems, rather than attempting to make existing building systems smarter.
\end{abstract}

Keywords: Adaptable; Housing; Skin; Robotics; Pneumatic

\section{Introduction}

In architecture, "smart" and "adaptable" building systems are finding themselves more deeply engrained within the ethos of design and fabrication. Buildings, which can sense temperature, occupation, day lighting, and other variables, are allowing for the more efficient/consistent monitoring of energy as well as internal and external environmental conditions. Constant communication between building systems and off-site smart personal technologies are allowing for the continuous oversight and manipulating of internal conditions from abroad.

These systems though are built upon a platform of materials, fabrication methodologies and architectural frameworks that are, outdated, highly ridged and resistant to change. Building forms continue to be calculated as finite elements during the design phase, only accounting for a fixed set of known variables. Materials are chosen from a small known pallet of predefined industrial standards, limiting our project's level of customization, flexibility or innovation. These standardized architectural forms can then minimally adapt to pre-calculated environmental/ programmatic changes through the manipulation of secondary, complex and rigid mechanized systems, which allow for small and simplistic motions.

\section{Japanese prefab housing}

In countries such as Japan where robotics and computational systems have been highly integrated into the design, fabrication and human interface of new housing, we witness higher levels of quality, customization as well as user and environmental interface. Robust software's such as those designed and utilized by some of the countries largest home manufactures, allow for the quick and efficient modification and modularization of building designs from both in-house designers and outside architects. These new technologies allow for the process of conceptualizing, designing, and constructing of small housing to be measured in days and weeks, rather than months or years.

With building elements robotically fabricated in controlled environments, digitally labeled and moved on-site with simplified instructions, once complex construction processes are now highly simplified allowing for the utilization of lower skilled laborers without the concern of a lower finish quality.

Although the construction industry in Japan is highly mechanized and data oriented, it is still highly based upon the refinement of centuries old materials and construction methodologies. Buildings although precise and spatially efficient, are still unable to adapt to Tokyo's rapidly evolving urban pace. With building life spans of only around 30 years, planning for specific conditions today does not guarantee that they will be efficient or relevant tomorrow.

With the volatility of today's environmental and urban conditions, we must learn to create buildings and data systems that are rapidly deployable, accessible, physically flexible and have the ability to rapidly adapt. Learning from the implementation of the complex data and fabrication methodologies in Japan, the One Day House aims to create an efficient and innovative system for the rethinking modern housing construction. 


\section{Design intelligence}

This paper looks at the creation of adaptable and intelligent architectural systems through the design and implementation of innovative materials and methodologies for "soft" building construction. Rather than attempting to make previous design methodologies "smarter", this project looks at design intelligence as a process that goes beyond the creation of so called "smart" artifacts and re-examines the entire process of design.

The intelligence of the building must become more deeply integrated into all aspects of design conception ranging from: How building codes are written within governments, to financial institutions and their policies for funding innovative projects, to existing and innovative materials/labor markets, through the design process, and finally down to the artifacts ability to physically adapt, and learn from its daily interactions with its inhabitants and the environment.

Through the rethinking of a typical "dwelling" as a starting point, we will reconceive the basic necessities of what is essential for living, as well as how the process of design and the finished artifacts can better adapt to their surrounding environments. Utilizing robotic on-site manufacturing, smart + adaptable + soft skin materials such as reinforced ETFE and intelligent infrastructure with embedded robotic systems, we will attempt to create not only building systems which can learn and adapt to its inhabitants and surroundings, but also create a robust market driven system which has the ability to adapt to our ever changing environmental, governmental and financial systems.

Through a series of explorations in rapid emergency housing prototypes, this paper will investigate the potential of highly flexible skin materials such as ETFE combined with integrated robotics to create a new typology of adaptable, rapidly constructed, high quality, low-cost and permanent housing skin systems. These explorations will push a series of four design strategies: 1) The re-evaluation of current spatial needs within typical housing. 2) The development of inflatable skin systems by pushing the boundaries of fabric modeling software's such as Meliar's MPanel plug-in for Rhino 3d. 3) The embedding of intelligent robotics within the facade system, allowing for a skin with the ability to adapt to ever changing internal and external conditions. 4) The evaluation and testing of temporary, onsite robotics for rapid fabrication and customization. Through these explorations this research will examine the feasibility of adaptable, inflatable, ETFE skin systems on small-scale rapidly prototyped housing systems.

\section{Simplified Systems}

Current prefab housing systems such as Sekisui's successful HEIM system, although composed of a series of simplistic modules, contain vast amounts of overall complexity. To allow for high levels of individual customization, Sekisui houses a massive 300,000 potential parts within the housing production line. Each individual house contains around 30,000 individual parts that must be correctly selected, organized, and installed robotically utilizing their HAPPS system (Furuse \& Katano, 2007).

Although extremely robust and efficient, the sheer complexity and high part count makes this typical methodology of housing prefabrication nearly impossible during the high demand, low cost needs following a natural disaster. Currently limited by traditional wood and steel construction methods, a successful delivery system for high quality emergency housing will require the creation of completely new prefabrication methodologies where individual elements are minimized, modules gain embedded intelligent systems and are fabricated as flexible unique elements on-site.

\section{Rethinking Modular}

To create a truly innovative and feasible system for rapid, custom and high quality modular emergency housing, our existing process of design and fabrication must be re-imagined. Large centralized factories must be replaced with small, mobile and agile on-site robotic fabrication shops that can been seen in prototypes like R-O-B (Gramazio \& Kohler,2014). Rather than utilizing known networks of small pre-defined shared parts in an attempt to create unique conglomerations, the One Day House begins fabrication at a larger module level. Through the utilization of the Rhino $3 \mathrm{~d} /$ Grasshopper computational interface, formal enclosures are continuously designed, manipulated, structurally + environmentally thickened and subdivided into large unique modules which are then fabricated of lightweight materials and aggregated on-site. The aggregation of larger lightweight modules allows for the minimization of complex units, and the easy implementation of integrated skin systems and infrastructure.

The One Day House is broken down into three unique, large, and simple components: Thickened floor, Condensed Core and Adaptable Surfaces.

\section{Thickened floor}

An elevated and thickened wet floor system houses flexible storage and furniture for the inhabitants, water storage, filtration and

Figure 2: One Day House v.1.3 exploded perspective, Created by Andrew John Wit.

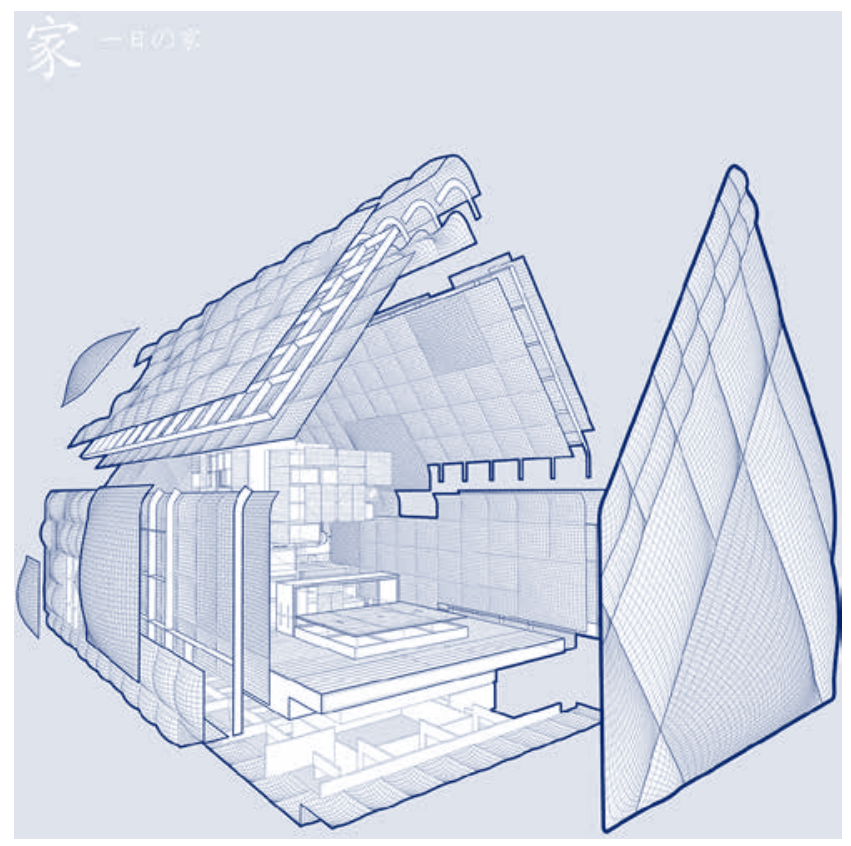


the composting sewage infrastructure. Structured upon an open composite framework and wrapped in recycled, local or fast growth materials on the interior and lightweight insulated ETFE on the exterior facades, the thickened floor system houses all necessary ridged infrastructure while creating a warm atmosphere within the dwelling. The flexible floor system allows inhabitants the ability to embed custom furniture with the ability to be reconfigured or hidden away, creating the appearance of more rooms while using less enclosed space.

\section{Condensed Core}

Linked directly into the wet infrastructure of the thickened floor is a flexible core. Compressed into a 7' x 7' x 9' volume, the Condensed Core houses all necessary living, data and electrical systems. Created through a rigorous series of design studies, the core's composite structure was derived through a series of simplistic structural folds, which minimize material thicknesses as well as allow for the formation of integrated multi-use spaces. Examples of this can be seen in areas such as the full bath, where the short enclosing wall continuously folds vertically. This structural folding allows for the creation of open storage in the bathing area while forming a compact stair within the storage wall that creates easy access to the core's top surface.

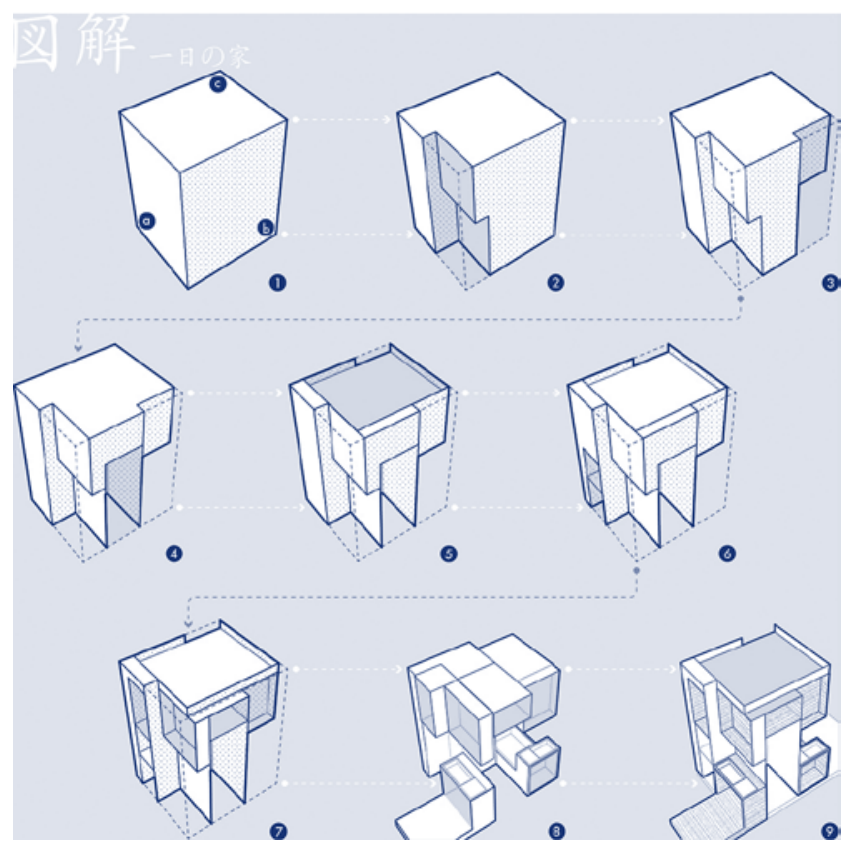

Figure 3: Core folding process, Created by Andrew John Wit.

In addition to creating the main clean + work spaces in the dwelling, the thin folding structure also encloses the data + energy infrastructure while creating a rigid framework in which a highly user customizable infill system can be attached.

In a small floor ventilated cavity between the toilet and storage space resides the dwelling's data, intelligent control center and partial energy storage systems. Energy collected through the exterior skins flexible solar cells is transferred to the core's batteries where the energy is then transmitted for localized use or sent to additional storage in the residents hybrid automobile battery systems. High-voltage systems found in kitchen items are directly linked to the intelligent core simplifying energy distribution complexity. Through the use of wireless phones, data and a further integration of short-range wireless energy transfer systems, complex infrastructural systems are nearly eliminated, lowering the cost and complexity of construction.

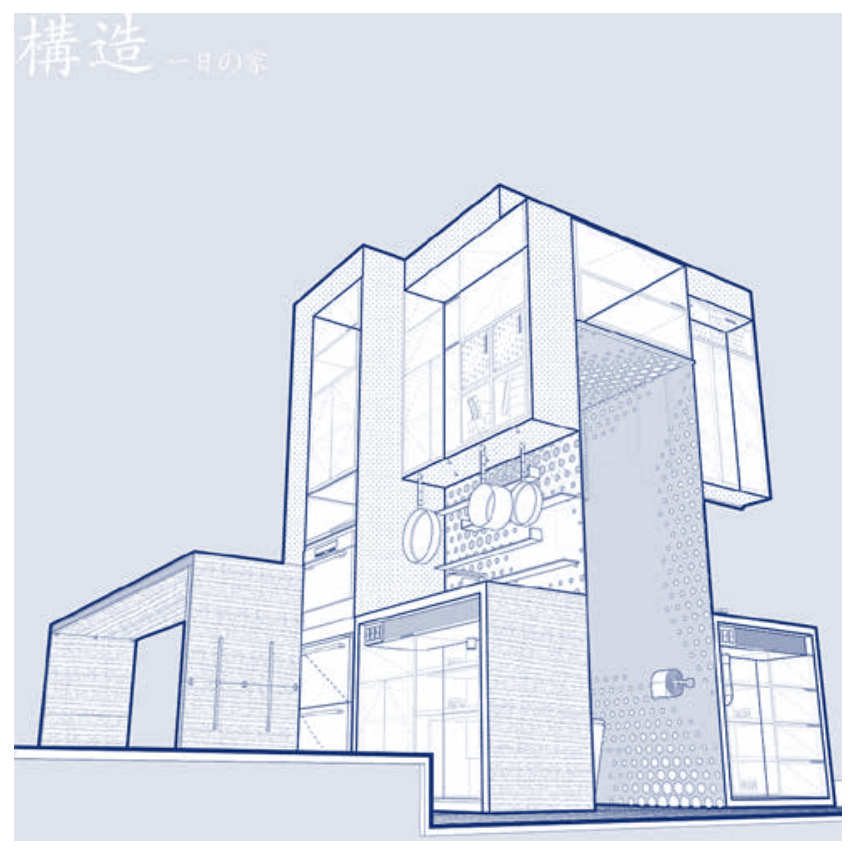

Figure 4: Condensed core with infill, Created by Andrew John Wit.

Where as the core's structural form remains rigid after completion, the infill system is highly flexible. Developed as an interactive plug and play Grasshopper interface, individuals are able to easily create unique infill storage systems based on their own needs and preferences. Built upon a simple packing system, size, location, orientation and material are all easily chosen and manipulated through the use of a series of sliders. Once a desired configuration and material pallet are chosen, the data is directly sent for fabrication. Material selection is highly flexible, able to accommodate local materials as well as materials which are easy to acquire after disaster situations. Requiring no additional hardware to attach into the core, the custom designed infill simply slides and stacks into the existing composite frame allowing the occupant the opportunity to reconfigure or replace individual units as needs or preferences change.

\section{Adaptable Surfaces}

Robotically controlled systems and smart materials are finding themselves ever more engrained within the evolving ethos of architecture. Although technological integration has begun to appear in applications such as reactive facades and environmental systems such as seen in the ITKE's Flectofin project (Lienhard, 2012), greater 
design interest has been focused on the integration of robotics into the process of architectural fabrication.

Within the automobile industry the BMW GENA (Bangle, 2008) has shown us a potential future for smart, soft, robotic skin systems. With its introduction of an engineered soft water resistant skin, the once complex conglomeration of materials, panels and gaskets becomes simplified into a flowing continuous skin. Wrinkling and stretching rather than splitting, the skin no longer remains a static element. Rather it is allowed to deform and change based on the needs and wants of the user.

Just as with automobiles, housing deal with continuously varying internal \& external environmental conditions. As with the BMW GENA, it is necessary to re-imagine the functionality and materiality of our buildings skin. With energy collection, water harvesting, natural ventilation and adapting to uncertain climatic conditions extremely important in rebuilding post-disaster, creating skin systems which can adapt over time to maximize optimal surface conditions is a necessity. Current building materials and systems restrict the exterior skin to singular final forms, which may or may not successfully sustain these conditions over time.

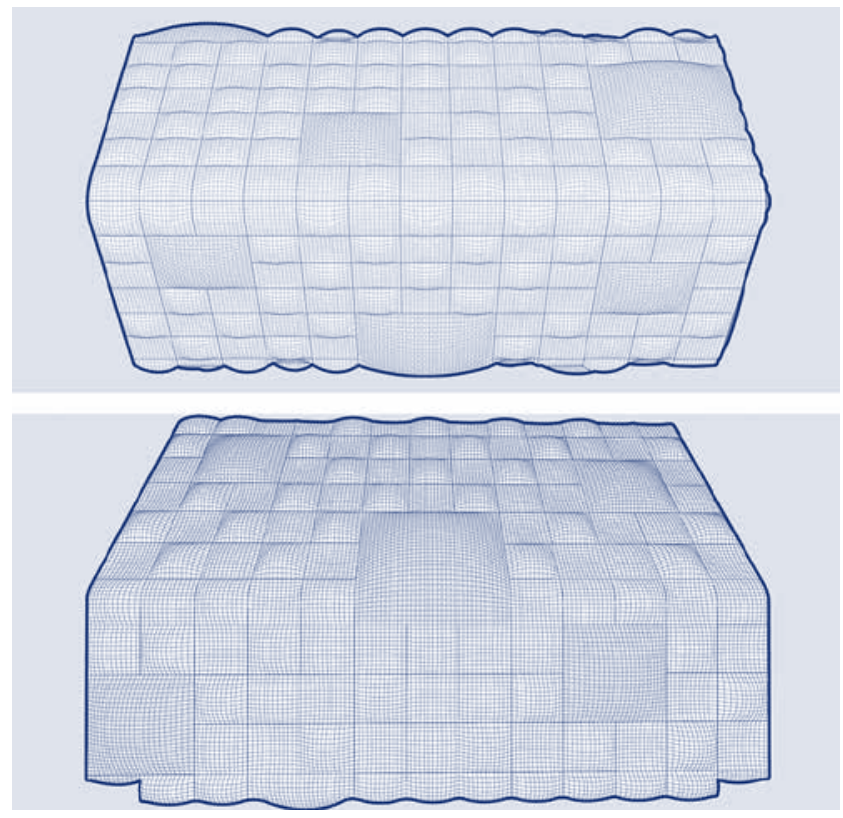

Figure 5: Adaptable Skin V.1.3, Created by Andrew John Wit.

The "One Day House" investigates adaptive façade systems both as a method of mediating environmental conditions and as an opportunity for design. Formal adaptability has been investigated through two systems: 1) A ridged modular composite structure joined with a flexible hinge and 2) A kinetic façade system consisting of a reinforced ETFE pillow system which wraps the structure. Where as the module's flexible joints allow for the deformation of the overall structure for large external forces, the kinetic façade allows for the dwelling to regulate internal temperature, as well as energy \& water harvesting.

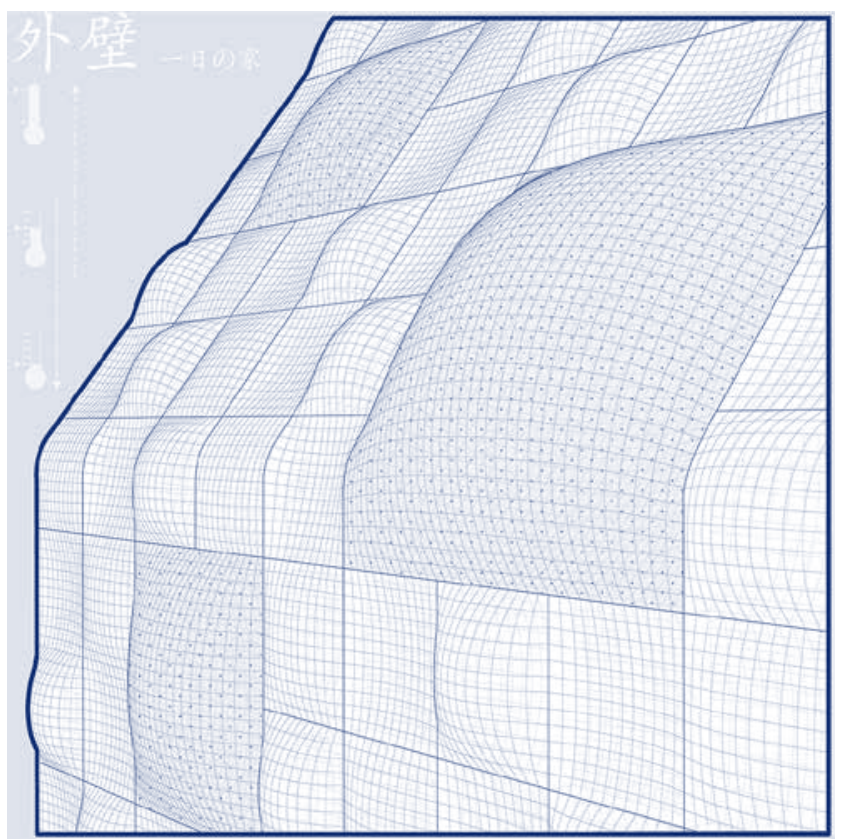

Figure 6: Adaptable Skin V.1.3, Created by Andrew John Wit.

\section{Parametric Skin:}

The design and parametric control of the active façade system was created through the integration of the MPanel \& Kangaroo physics within the Rhino $3 \mathrm{~d}$ design environment. Initial design inputs such as material, tension, internal air pressure, etc., were created within the MPanel software. The output, a static formal tensile skin was then linked directly into kangaroo where the model was parameterized creating a model whose skin had the ability to continuously update based on internal and external design constraints. After a series of predicted constraints were tested within the Kangaroo environment, the model was then checked again in MPanel to verify material stresses and tolerances. The resultant is the adaptable skin V.1.3 that is currently in further development.

\section{Conclusion}

The "One Day House" is the first step in the process of rethinking rapid emergency housing through the integration of smart materials and intelligent adaptable systems. Through the simplification of a new materials palette and the creation of smarter fabrication methodologies, the author aims to offset the higher upfront costs of materials and advanced technologies through the minimization of materials, labor, infrastructure and overall long-term environmental and energy costs.

Focusing not on formal architectural gestures but rather on how "form" can be generated through environment, the project will continue to question current notions of the correct "form" of buildings through the testing of materials and systems who's "form" has the ability to adapt to their internal and external environments.

With the initial studies focused on the developing of a design, materials, systems as well as parametric design language for the prototype continued research will now delve further into robotic 
fabrication. Through the creation and testing of a on site fabrication workflow as well as full-scale material and design prototypes, the author will further test the cost, complexity and feasibility of the proposed housing prototype which could pave the way for future permanent post-disaster housing.

\section{Acknowledgments}

Special thanks to the following contributors throughout the development of the One Day House:

Meliar Design for the use of their tensile membrane modeling software MPanel throughout our continuing research.

Ball State University for its provided funding through a series of internal grants, as well as for their acquisition of our industrial robot which will help further this research through a series of larger scale working prototypes.

\section{References}

Baldwin, C.Y. \& Clark, K.B. (2000). Design Rules, Vol.1: The Power of Modularity. Cambridge, MA: MIT Press.

Bangle, C. (2008). BMW group: Gina Light Vision Model. Pasajes de arquitecturea y critica. No. 101, p.32.

Barlow, J. \& Ritsuko, O. (2005). Building mass customized housing through innovation in the production system: Lessons from Japan. Environment and Planning, A37(1), 9-20.

Biloria, N. \& Sumini, V. (2009). Performative building skin systems: A morphogenomic approach towards developing real-time adaptive buildings skin systems. International Journal of Architectural Computing, Vol..7 no. 4, 643-676.

Cardoso, D., Michaud, D. \& Sass, L. (2007). Soft Façade: Steps into the definition of responsive ETFE façade for high-rise buildings. "Predicting the future", 25th eCAADe conference proceedings, 567-573.
Furuse, J \& Katano, M. (2006). Structuring of Sekisui HEIM automated parts pickup system (HAPPS) to process indivisual floor plans. The 23rd International Symposium of Automation and Robotics in Construction 2006, 352-356.

Gramazio, F., \& Kohler, M. (2014). Made by Robots: Challenging Architecture at the Large Scale, AD Wiley.

Gross, J. \& Green, K.E. (2012). Architectural robotics inevitably. Interactions. Volume XIX.1, N.Y.

Hensel, M., Menges, A. \& Weinstock, M. (2010). Emergent Technologies and Design. NY: Routledge.

Khoo, C.K. \& Salim, F. (2013). Responsive Materiality for morphing architectural skins. Proceedings of the 33rd Annual Conference of the Association for Computer Aided Design in Architecture (ACADIA), 243-252.

Kieran, S. \& Timberlake, J. (2004). Refabricating Architecture. N.Y.: McGraw Hill.

Lienhard, J., Schleicher, S., Poppinga, S., Masselter, T., Milwich, M., Speck, T., \& Knippers, J. (2011). Flectofin: a hinge-less flapping mechanism inspired by nature. Biomim. 6, 045001.

Partridge, K. (2010). Robotics. The Reference Shelf. Volume 81, N.Y.

Piroozfar, A.E.P \& Piller, F.T (2013). Mass Customisation and Personalisation in Architecture and Construction. N.Y, N.Y: Routledge.

Sach, E. (2008). Synthesis of Form, Structure and Materials: Design for a Form-Optimized Lightweight Membrane Construction, Silicon + Skin: Biological Processes and Computation. Proceedings of the 28th Annual Conference of the Association for Computer Aided Design in Architecture (ACADIA), Minneapolis, MI, 200-207.

Silver, M. (2013). Off-road City. ACADIA 13: Adaptive Architecture. Proceedings of the 33rd Annual Conference of the Association for Computer Aided Design in Architecture (ACADIA), Cambridge, Ontario, Canada, 445-446. 